\title{
Como as tecnologias assistivas para acesso a computadores podem ajudar as pessoas
}

\section{com lesão medular?}

How can assistive technologies for accessing computers help people with spinal cord injuries?

¿Cómo pueden las tecnologías de asistencia para acceder a las computadoras ayudar a las personas

con lesiones de la médula espinal?

Recebido: 24/08/2021 | Revisado: 29/08/2021 | Aceito: 17/01/2022 | Publicado: 19/01/2022

Valéria Baldassin

ORCID: https://orcid.org/0000-0002-8664-8101 Associação das Pioneiras Sociais, Brasil E-mail: valbaldassin@gmail.com

Helena Eri Shimizu

ORCID: https://orcid.org/0000-0001-5612-5695 Universidade de Brasília, Brasil

E-mail: Shimizu@unb.br

\begin{abstract}
Resumo
Objetivo: analisar a percepção de indivíduos com tetraplegia por lesão medular sobre a influência do uso de recursos de tecnologia assistiva (TA) para acesso a computadores no seu cotidiano. Método: estudo com abordagem qualitativa, tendo como referencial teórico-metodológico, a Grounded Theory ou Teoria Fundamentada nos Dados. Foram realizadas 27 entrevistas semiestruturada com indivíduos com tetraplegia assistidos na Rede SARAH de Hospitais de Reabilitação de Brasília. Os dados foram analisados com o auxílio do software ATLAS.ti. Resultados: As TA para acesso a computadores possibilitam a diminuição da vulnerabilidade e a ampliação da autonomia das pessoas portadoras de lesão medular grave, em diversas perspectivas, bem como a sua reinserção social e no trabalho. Conclusão: É necessário incluir nos serviços de atendimento as pessoas com lesão medular grave, a possibilidade de uso TA, desde o inicio do tratamento, com devido acompanhamento de profissionais qualificados para orientar o uso dos mesmos. Assim sendo, deve-se reconhecer o direito de acesso a TA a essas pessoas para melhoria da qualidade de vida e alcance da dignidade humana. Torna-se um imperativo ético garantir o acesso dessas pessoas aos recursos de TA, através da ampliação de políticas públicas intersetoriais, que não pode estar apartado de outros esforços empreendidos pelo poder público, no sentido de qualificar a vida social desses cidadãos.
\end{abstract}

Palavras-chave: Lesão medular; Tecnologia assistiva; Autonomia; Bioética.

\begin{abstract}
Objective: to analyze the perception of people with quadriplegia due to spinal cord injury about the influence of the use of assistive technology (AT) resources to access computers in their daily lives. Method: Qualitative approach, the Grounded Theory. Twenty-seven semistructured interviews were performed with individuals with quadriplegia assisted in the SARAH of Rehabilitation Hospitals of Brasília. The data were analyzed with ATLAS.ti software. Results: AT for access to computers makes it possible to reduce vulnerability and expand the autonomy of people with severe spinal cord injury, in different perspectives, as well as their social and work reintegration. Conclusion: that it is necessary to include in the care services people with severe spinal cord injury, the possibility of using AT, from the beginning of treatment, with proper monitoring of qualified professionals to guide their use. Therefore, these people's right of access to AT should be recognized in order to improve their quality of life and achieve human dignity. It becomes an ethical imperative to guarantee these people's access to AT resources, through the expansion of intersectorial public policies, which cannot be separated from other efforts undertaken by the government to improve the social life of these citizens.
\end{abstract}

Keywords: Spinal cord injury; Assistive technology; Autonomy; Bioethics.

\section{Resumen}

Objetivo: analizar la percepción de individuos con tetraplejía por lesión medular sobre la influencia del uso de recursos de tecnología asistida para acceso a computadoras en su cotidiano. Método: estudio con abordaje cualitativo, teniendo como referencial teórico-metodológico, la Grounded Theory o Teoría Fundamentada en los Datos. Se realizaron 27 entrevistas semiestructuradas con individuos con tetraplejia asistidos en la Red SARAH de Hospitales de Rehabilitación de Brasilia. Los datos fueron analizados con la ayuda del software ATLAS.ti. Resultados: La TA para el acceso a las computadoras permite reducir la vulnerabilidad y ampliar la autonomía de las personas con lesión medular severa, en diferentes perspectivas, así como su reinserción social y laboral. Conclusión: Es necesario incluir 
en los servicios de atención a las personas con lesión medular severa, la posibilidad de utilizar TA, desde el inicio del tratamiento, con el debido seguimiento de profesionales calificados para orientar su uso. Por tanto, se debe reconocer el derecho de estas personas al acceso a la TA para mejorar su calidad de vida y alcanzar la dignidad humana. Se convierte en un imperativo ético garantizar el acceso de estas personas a los recursos de TA, a través de la expansión de políticas públicas intersectoriales, que no pueden separarse de otros esfuerzos que realiza el gobierno para mejorar la vida social de estos ciudadanos.

Palabras clave: Lesión de la médula espinal; Dispositivos de autoayuda; Autonomía; Bioética.

\section{Introdução}

A incidência de lesão medular traumática no Brasil é desconhecida, porém, estima-se que na América do Sul seja de 25 casos, e a mundial 23 casos por milhão de habitantes ao ano (Lee et al., 2014).

A imobilidade e a consequente não funcionalidade do corpo conferem vulnerabilidade específica aos indivíduos com tetraplegia, que repercutem em sua corporeidade, independência física e autonomia (Baldassin et al, 2018). Embora a pessoas com lesão medular grave apresentem, em sua maioria, sua integridade cognitiva preservada e capacidade de efetuar escolhas, podem se sentir incapazes de se autogovernar, o que provoca um efeito negativo em suas autonomias (Rezende et al., 2018).

As pessoas com deficiências, em geral, estão entre as mais estigmatizadas, por vezes, como consequencia das suas condiçoes físicas debilitadas, mais pobres e com os níveis de escolaridade mais baixos (United Unions, 2011). Portanto, a compreensão da vulnerabilidade de pessoas com tetraplegia no Brasil deve se pautar, tanto nos aspectos inerentes às sequelas da lesão, que as expõem à vulnerabilidade individual, como nos determinantes sociais, culturais e econômicos, que potencializam essa vulnerabilidade. Destarte, o aumento da expectativa de vida de pessoas com lesão medular tem resultado em maior participação no seu próprio cuidado (Gómara-Toldrà et al., 2014), sendo este um dos principais objetivos do processo de reabilitação integral (Schwartz et al., 2018), no qual deve se incluir a utilização contextualizada da tecnologia assistiva, que engloba recursos, práticas e serviços que promovam melhoria da funcionalidade corporal (Decreto $\mathrm{n}^{\mathrm{o}} 10.645$, 2021).

Nesse processo de reabilitação, o treinamento das pessoas com lesão medular para lidar com as mudanças físicas, psicológicas e sociais torna-se essencial. Há que se trabalhar com o significado global das mudanças, que inclui a interpretação da experiência estressora, mais precisamente causada pela limitação física abrupta, bem como a oferta de estratégias ou recursos para motivador mudanças e ações e para que a pessoa possa planejar o futuro (Bailey et al.,2016; Litooji et al., 2016; Silva et al., 2021 ).

Nessa lógica, as tecnologias assistivas, especialmente alguns softwares e hardwares para uso de computadores podem representar a única possibilidade de acesso à informação, ao trabalho e a socialização para essas pessoas com lesão medular, favorecendo assim, o exercício de sua autonomia. Esses recursos são oriundos de recentes avanços tecnológicos e podem contribuir para facilitar diversos aspectos do desempenho humano, desde tarefas básicas de autocuidado até atividades profissionais, pois podem ser adaptados ao grau de incapacidade, portanto com grandes potencialidades para ajudar no resgate da autonomia.

Logo, o acesso, especialmente as tecnologias para acesso a computadores, doravante TA, porta um inegável caráter bioético constituído pela confluência de princípios como: justiça na distribuição dos benefícios das novas tecnologias, respeito às vulnerabilidades humanas específicas e proteção das pessoas com limites de autonomia (Baldassin, et al, 2018; Unesco, 2005).

As especificidades da expressão dos limites da autonomia em indivíduos tetraplégicos impõem a necessidade de compreender três dimensões da autonomia: a decisória, a de informação e a executiva ou de ação (Beauchamp \& Childress, 2013; Seoane, 2010). A autonomia decisória está centrada na liberdade de escolha do indivíduo, a autonomia da informação consiste na sua capacidade de estabelecer e controlar suas informações pessoais e, por fim, a autonomia executiva ou 
funcional, se refere à liberdade de ação ou abstenção, que pode estar significativamente comprometida no indivíduo tetraplégico, dada sua condição de imobilidade. Assim sendo, a autonomia deve ser pensada como uma necessidade, um valor e um dever, além de possuir caráter ontológico, moral e normativo (Beauchamp \& Childress, 2013; Unesco, 2005).

O conceito contemporâneo de autonomia tem duas origens principais com diferenças marcantes entre elas. A primeira na noção de autonomia relacional, que parte das ideias de dignidade, liberdade e responsabilidade individuais, defendidas por Kant, para quem as escolhas individuais devem considerar seus efeitos sobre a coletividade (Habermas, 2003), e a segunda na noção de liberdade subjetiva, desenvolvida pelos utilitaristas ingleses, tais como Stuart Mill e Jeremy Bentham, vinculando autonomia à capacidade e ao direito de uma individualidade singular, separada da comunidade, seguindo as próprias preferências (Habermas, 2003; Seoane, 2010). Entretanto, comum a ambas as concepções de autonomia, são as exigências para que uma escolha seja considerada autônoma: intencionalidade, compreensibilidade e ausência de interferência coercitiva (Habermas, 2003).

A autonomia compreendida de forma relacional parece mais adequada para tratar nosso problema, pois não se trata somente da liberdade dos indivíduos, mas também de condições para sua auto-realização como um todo, uma vez que a maximização da autonomia de indivíduos tetraplégicos necessita de intervenção do Estado para garantir condições que tornem seu exercício e desenvolvimento possíveis.

Apesar das evidências sobre os benefícios da TA por pessoas com deficiências físicas graves (Fabrin et al., 2016; Silva et al., 2021), ela não é amplamente utilizada devido ao custo elevado, à carência de oferta de mercado e à escassez de políticas públicas voltadas para esse tema, razões que se agravam ainda mais no contexto de países em desenvolvimento. A dimensão bioética envolvida na proteção à vulnerabilidade e na superação das iniquidades que limitam a autonomia do indivíduo tetraplégico envolve, portanto, responsabilidades da sociedade, em especial familiares e profissionais, e do próprio Estado.

Desta feita, a demonstração da importância da TA pelos próprios indivíduos tetraplégicos pode colaborar para desenvolvimentos de programas sociais e políticas públicas para melhoria do acesso. Apesar disso, são escassos os estudos desenvolvidos no Brasil que avaliam a percepção de pessoas com tetraplegia sobre a influência do uso de TA em sua autonomia e na reintegração social. Assim sendo, este estudo se justifica devido à necessidade de avançarmos nos conhecimentos e argumentos necessários para possibilitar maior autonomia às pessoas portadoras de lesão medular grave por meio da TA para acesso a computadores. O estudo teve como objetivo analisar a percepção de indivíduos com tetraplegia por lesão medular sobre a influência do uso de recursos de TA para acesso a computadores no seu cotidiano.

\section{Metodologia}

Estudo qualitativo, fundamentado na Grounded Theory (GT) desenvolvido pro Glaser e Strauss, que permite focar no contexto, na complexidade e análise situacional do fenômeno(Charmaz, 2009). Esta metodologia permite construir indutivamente uma teoria a partir da coleta, codificação e comparação simultânea e sistemática dos dados (Charmaz, 2009). Foi realizado com pessoas que sofreram lesão medular grave atendidas na Rede SARAH de Hospitais de Reabilitação, unidade Brasília, centro de referência nacional, que possui como meta devolver ao cidadão os impostos pagos através de atendimento público de qualidade, humanizado e com tecnologia de ponta.

Os critérios de inclusão foram: indivíduos maiores de 18 anos, com lesão medular cervical traumática, lesão motora completa e usuários de recursos de TA. Os critérios de exclusão foram: indivíduos menores de 18 anos e lesão encefálica associada.

A amostra foi composta por 27 indivíduos, que participaram de entrevistas semiestruturadas, que os indagavam sobre os tipos de recursos de TA utilizados para acessar computadores, suas percepções sobre autonomia e as repercussões do 
acesso ao computador em suas vidas, sobretudo nas atividades que realizavam em suas rotinas. Com base na literatura sobre entrevista que tratam de mudança global (Bailey et al., 2016; Litooiji et al., 2016), utilizamos os seguintes tópicos: o que aconteceu? Quais foram as mudanças? O que não mudou? Com a lista de tópicos em mente como uma diretriz, o entrevistador seguiu o fluxo natural da conversa. Resumindo e reformulando o que ouviu, a pesquisadora testou constantemente suas suposições durante a entrevista, chegando às camadas mais profundas do significado global (Bailey et al., 2016; Litooiji et al., 2016). Os participantes puderam falar livremente sobre as experiências vividas, sem interrupções, o que possibilitou clima de empatia com o pesquisador. Em média as entrevistas duraram cerca de uma hora. Foi realizado estudo piloto e o encerramento das entrevistas baseou-se na saturação dos conteúdos obtidos nas entrevistas (Charmaz, 2009). Adicionalmente, o entrevistador registrou as observações feitas antes, durante e após as entrevistas, com detalhes da situação da entrevista, ocasionalmente os aspectos não verbais.

Posteriormente, as entrevistas foram transcritas na integra. Na primeira etapa da análise, essas entrevistas foram inseridas no software ATLAS. ti, versão 2005, utilizado para análise qualitativa de grandes quantidades de dados. A segunda etapa, chamada de codificação aberta, consistiu em estabelecer uma relação lógica entre as categorias e os fragmentos das falas, respectivamente denominados pelo software por "codes" e "quotations". A terceira etapa, chamada de codificação axial, tem como objetivos classificar, sintetizar e organizar grandes montantes de dados e reagrupá-los de novas formas após a codificação aberta (Charmaz, 2019). Aqui as categorias e subcategorias foram integradas a partir da elaboração de conexões entre elas. Finalmente, na última etapa, foi feita a codificação seletiva, onde foram identificadas as categorias principais, limitando-se a codificar aspectos que pertençam à categoria central e às categorias associadas a ela de forma significativa(Charmaz, 2019).

O projeto foi aprovado pelo Comitê de Ética da Associação das Pioneiras Sociais, sob o registro ${ }^{\circ}$ CAAE 28727214.2.0000.0022.

\section{Resultados}

A maioria dos participantes era do sexo masculino, jovens, solteiros, sem ocupação e os recursos mais utilizados para acessar computadores foram: clipe palmar, adaptação para o dedo, touchmouse, haste para digitar com a boca e joysticks. Estes equipamentos possibilitam a manipulação do computador, que são indicados de acordo com as capacidades individuais das pessoas acometidas por lesão medular.

Apresentam-se, a seguir, as etapas percorridas na construção da pesquisa fundamentada (codificação aberta para construção das cetegorias iniciaias, codificação axial para apresensão das categorias principais e codificação final para a síntese da categoria central). 
Figura 1. Etapas da construção da Grounded Theory. Brasília-DF, 2021.

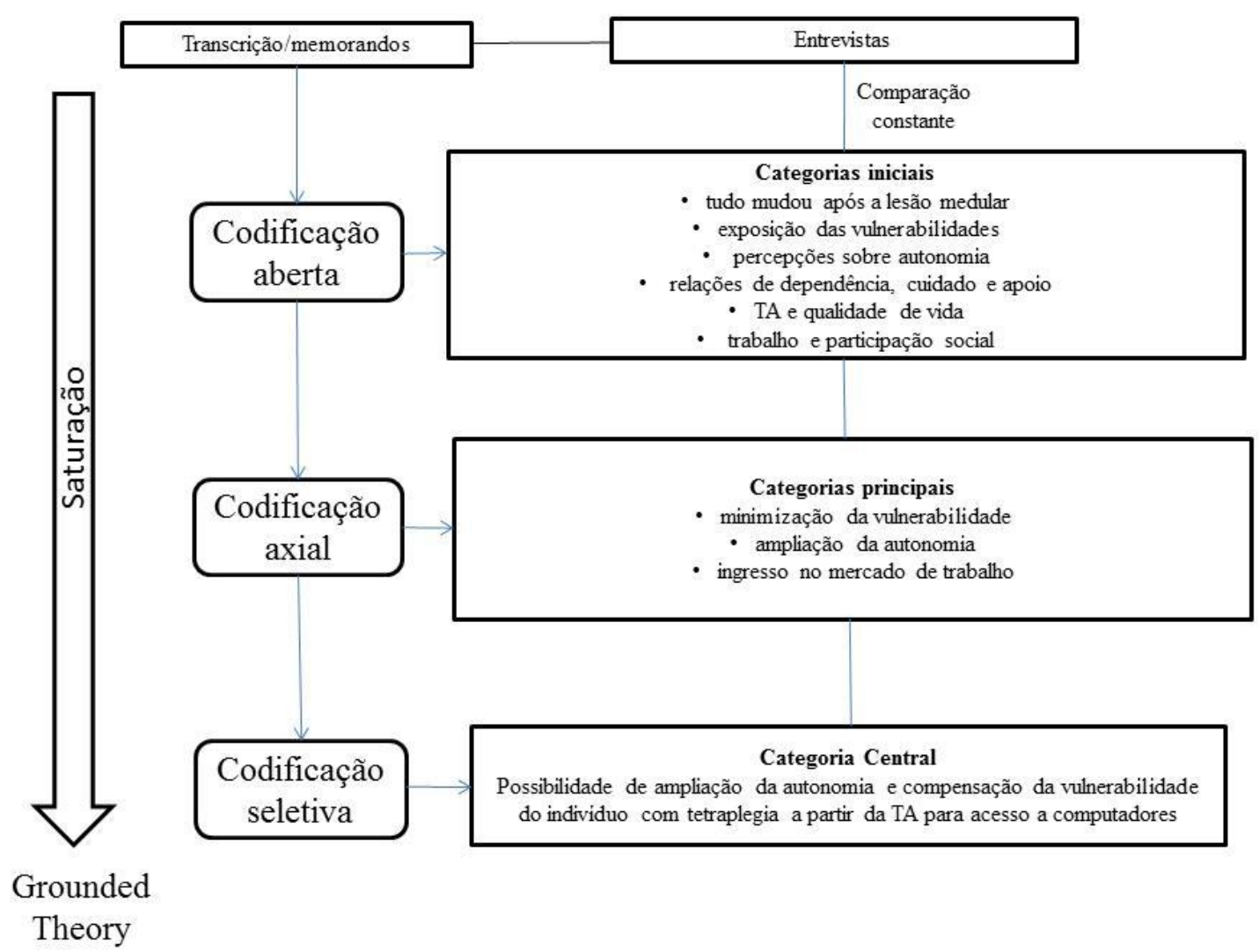

Fonte: Autores.

$\mathrm{Na}$ codificação aberta se destacaram seis categorias iniciais, de acordo com sua relação lógica, com a coerência que apresentavam entre si e com os objetivos do estudo, sendo elas: 1) tudo mudou após a lesão medular, 2) exposições sobre vulnerabilidade, 3) percepções sobre autonomia, 4) relações de dependência, cuidado e apoio, 5) tecnologia assistiva e qualidade de vida, 6) trabalho e participação social. A Figura 2 mostra as diversas mudanças significativas que ocorreram na vida após a lesão medular, e os sentimentos que aconpanharam o enfretamento desse momento. 
Figura 2. Mudanças e sentimentos após a lesão medular

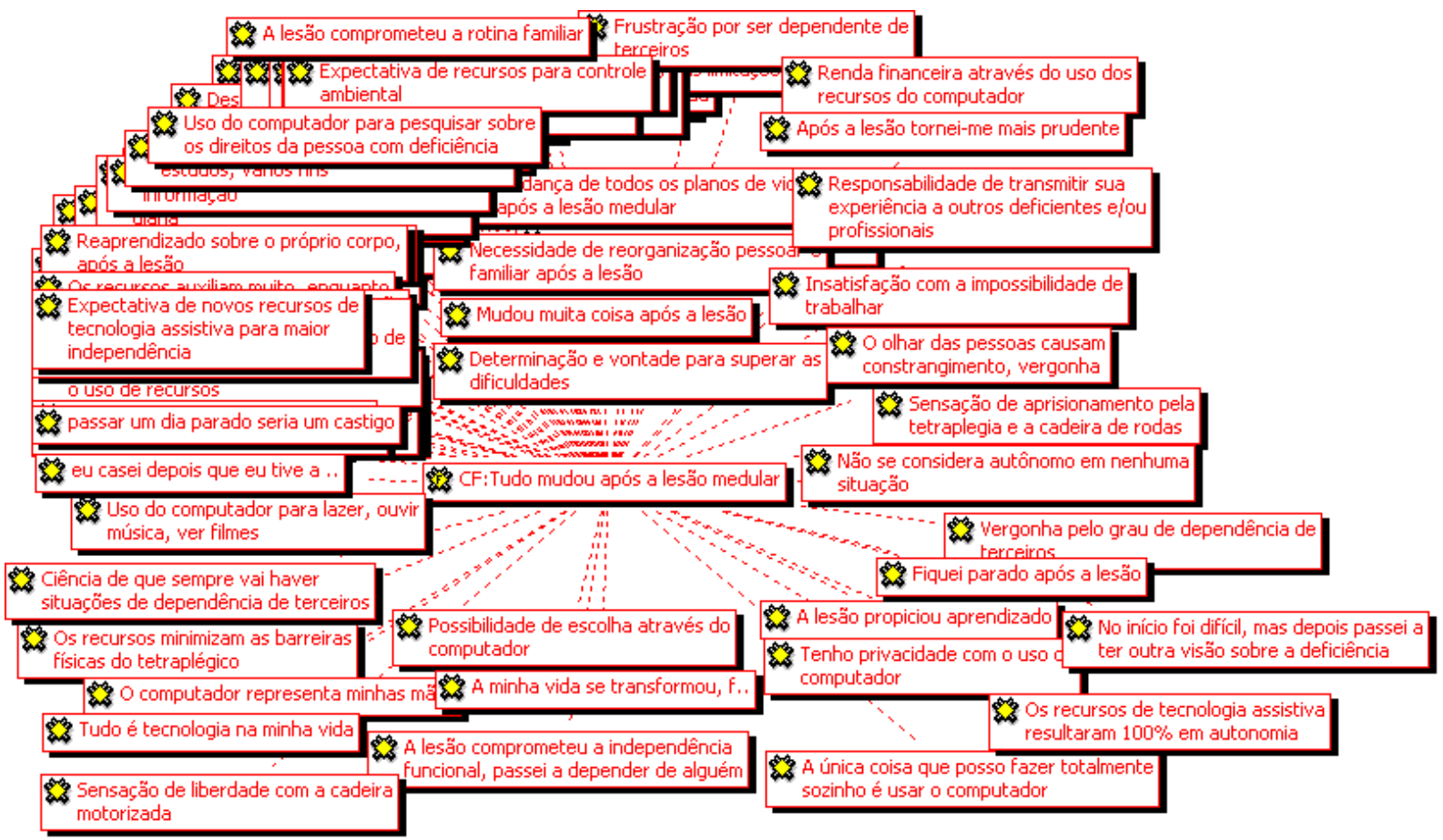

Fonte: Autores.

O sentimento de mudança brusca em suas rotinas após a lesão medular, especialmente relacionado ao impacto das perdas no período inicial pós-trauma, esteve presente nas falas de todos os entrevistados e foi vinculado a diversas esferas de suas vidas, especialmente à dependência física de terceiros para a execução de atividades da vida diária, como higiene, alimentação e transferências. A sensação de aprisionamento diante da tetraplegia e associada à dependência foi reportada por alguns participantes, assim como a necessidade de reaprendizagem sobre o próprio corpo. A condição de vulnerabilidade e falta de autonomia estava presente em vários contextos de suas vidas, dentre os quais se destacaram física/corporal, psicológica/emocional e social.

(...) desde pegar uma coisa com a mão, sair da cama, passar para a cadeira, ir ao banheiro sozinho, de noite se virar sozinho na cama, poder coçar a orelha, a cabeça (P22).

As relações de cuidado e apoio foram expressas de várias formas nas falas dos participantes e estiveram atreladas especialmente ao cuidado físico que demandavam e recebiam de seus familiares e cuidadores. A dependência de terceiros para a execução da maioria das atividades de vida diárias foi o aspecto mais negativo decorrente da lesão medular, segundo a maioria dos participantes.

A maioria dos entrevistados referiu que o uso das tecnologias assistivas resultou em melhor qualidade de vida, pelo fato de terem alcançado independência para a execução de várias atividades de vida diária com o uso de adaptações de vida diárias e interfaces de acesso a computadores. Associaram o sentimento de independência e de auto eficácia com qualidade de vida. O uso das TA para uso do computador possibilitou efetuar suas escolhas, interagir com outras pessoas, acessar informações e resultou na sensação de liberdade, felicidade e bem estar.

(...) Se estou no computador, não quero que ninguém veja aquilo, eu faço a minha escolha, li, terminei, desliguei, pronto. Antes não, se eu estava lendo algo, aí, alguém olhava e perguntava: você está lendo isso por quê?. Minha 
irmã que tinha que ficar conversando pra mim aí eu ficava com vergonha e hoje em dia não, ninguém precisa saber o que eu faço na internet, só eu" (P18).

Os entrevistados expuseram, ainda, suas dificuldades relacionadas ao ingresso no mercado de trabalho, e a participação social, pelo fato de não conseguirem se movimentar e por serem dependentes de outras pessoas para muitas tarefas.

Na codificação axial, as categorias iniciais foram reagrupadas e emergiram três categorias relacionadas às percepções dos participantes sobre as consequências da lesão medular e o uso da TA: 1) minimização da vulnerabilidade, 2) influência da TA na ampliação da autonomia e 3) possibilidades de ingresso no mercado de trabalho. Na figura 3 mostra-se os benefícios das TA na diversas dimensões da qualidade de vida das pessoas.

Figura 3.

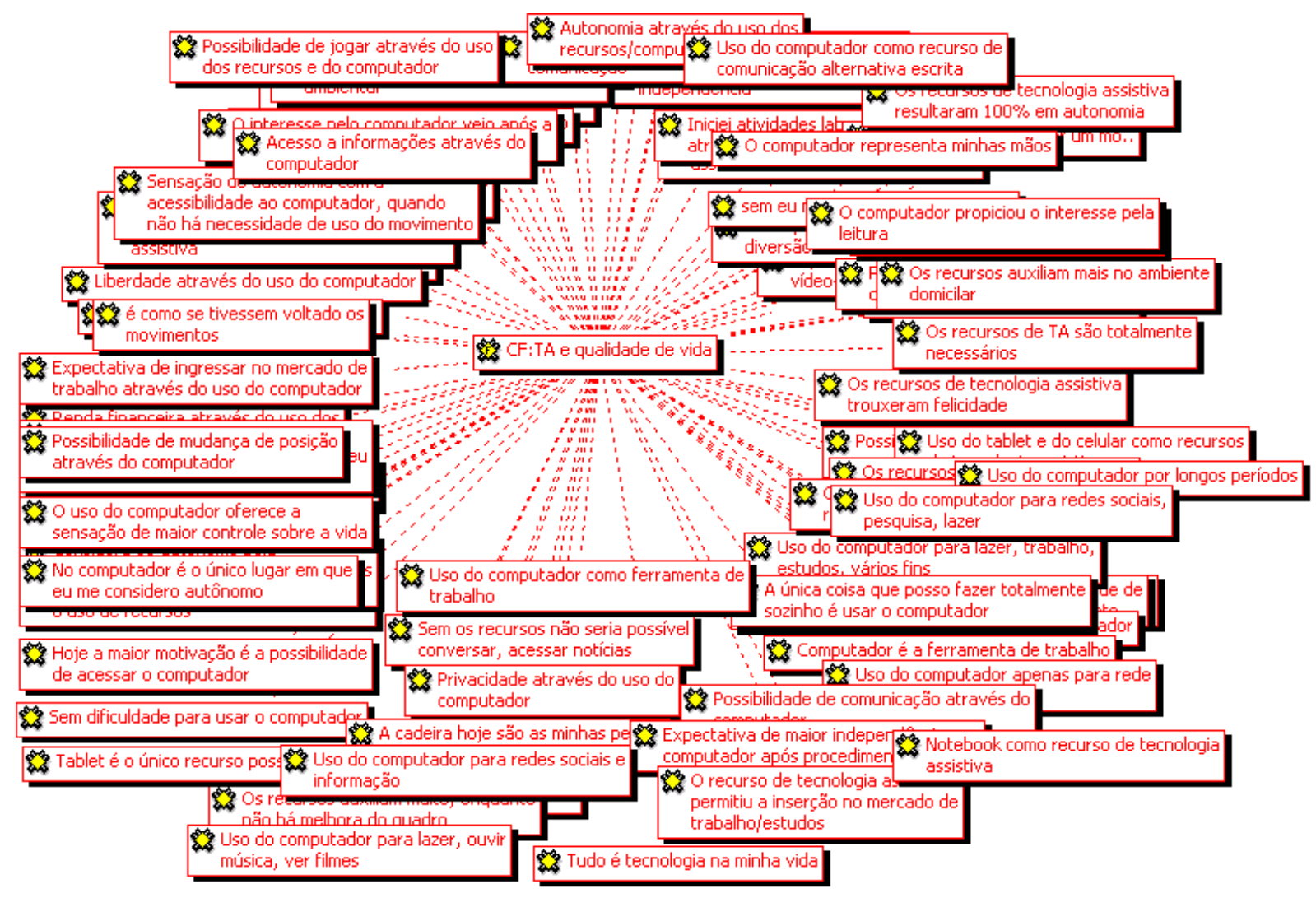

Fonte: Autores.

A maioria dos entrevistados afirmou que o incremento da qualidade de vida foi associado ao uso do computador e o seus recursos como joysticks e várias adaptações que possibilitaram a prática de algum hobby, o acesso a jogos, a redes sociais, a informações, auxiliaram na execução de atividades escolares e laborais ou propiciaram simplesmente distração, como assistir a filmes e ouvir música.

Assim, a categoria: "minimização da vulnerabilidade através do uso de computadores" retrata o reconhecimento dos participantes dos benefícios dos recursos de TA em suas rotinas, na medida em que aliviaram o peso da imobilidade e viabilizaram o fazer algo por si mesmos, sem depender do outro.

"Acabou, agora não ando, não mexo as mãos, o que vou fazer? Eu faço, eu posso, eu tenho autonomia e isso (recurso de acesso a computadores) tornou a realização de grandes sonhos, de grandes conquistas” (P20). 
As representações expressas pelos participantes sobre autonomia foram variadas e se relacionaram principalmente à independência funcional. Alguns associaram autonomia à liberdade, privacidade, proatividade, comunicação, acesso à informação, escolha e tomada de decisão.

Alguns participantes evidenciaram o uso de tecnologias mais sofisiticadas, como o comando de voz, que permite outra forma de comunicação mais direta com outras pessoas, o que representa aumento do gradiente de autonomia.

(...) tudo o que for externo eu preciso de alguém, mas na hora que eu uso o computador eu estou interagindo diretamente, sem ter uma outra pessoa...isso para mim é autonomia, agora vou usar o software por comando de voz para escrever meu livro (P5).

O acesso à informação por meio da TA propiciou, para vários participantes, maior autonomia através do aprendizado e da capacitação, que lhes permitiram projetar suas vidas, reabastecendo-os de esperança, no sentido de seguir em frente. "Hoje eu sou bolsista da Associação dos Pintores com a Boca e com os Pés, se não fosse o computador eu não sei como eu estaria hoje" (P17).

Os depoimentos revelaram que diversas habilidades foram desenvolvidas por meio do uso dos computadores, possiblitando o retorno à vida laboral. Um dos entrevisados como o computador com acessórios específicos tem auxiliado na digitação de obras grandes como livros.

(...) O computador, as adaptações para pintar e a adaptação para o computador são minhas ferramentas de trabalho. Eu não faço ideia de como seria a minha vida sem essas coisas porque tudo o que aconteceu na minha vida vem daí, foi o primeiro passo, o meu contato com a adaptação da boca para digitar o meu primeiro livro, o segundo, o terceiro, tenho mais dois prontos. Agora eu estou coordenando oficinas com crianças e com senhoras no meu bairro" (P17).

Outro entrevistado mostrou que conseguiu reinserção no mercado de trabalho na área das mídias sociais.

(...)Depois que eи comecei a usar o mouse eи comecei a digitar mais, aí eu pude fazer minhas próprias palestras e com isso eu consegui um emprego. Eu sou responsável por atualizar informações de um site, facebook e twitter de uma empresa" (P2).

Todavia, os participantes revelaram que as pessoas com deficiências ainda são escassas as oportunidades no mercado de trabalho, especialmente em cidades pequenas.

(...) Eu já mandei muitos currículos na minha cidade, eu tenho bastantes cursos de computador, mas eles disseram que não estavam contratando em cidades do interior(P19).

(...)Vontade de trabalhar eu tenho daí, eu vou atrás vejo que é muito difícil e acabo desistindo porque eu moro numa cidade bem pequena, tem cursos à distância, mas as provas são presenciais, aí dificulta (P22).

Expressaram ainda a necessidade de meios para ampliar o conhecimento em informática, o que poderia contribuir para o retorno ao trabalho. "Acho que tem que ter mais investimentos, mais pessoas pensando nisso, ajudando a pensar nessa possibilidade que a gente que precisa" (P3).

Ademais, ficou implícita nas narrativas a carência de intervenções que garantam melhores condições de acessibilidade urbana, postos de trabalho adaptados, acesso a inclusão digital e participação social, pois esses participantes se sentiram privados de seu potencial e de condições estruturais básicas para realizar um de seus principais projetos de vida: o retorno ao trabalho. 
No contexto deste trabalho, a análise envolvendo o fenômeno da exacerbação da vulnerabilidade e comprometimento da autonomia do indivíduo tetraplégico foi o seguinte: Condição causal (lesão medular) $\Rightarrow$ Fenômeno (dependência funcional, exacerbação das vulnerabilidades e minimização da autonomia) $\Rightarrow$ Contexto (comprometimento motor grave, dependência para várias atividades, condições sociais desfavoráveis) $\Rightarrow$ Condições Intervenientes (dificuldades para execução de atividades em vários setores da vida) $=>$ Estratégias de ação/interação (promoção de acesso a recursos de TA para uso de computadores) => Consequências (possibilidade de ampliação da autonomia, da qualidade de vida e minimização das vulnerabilidades).

Finalmente a codificação seletiva apontou para uma categoria central, construída a partir da reunião de categorias sobre o pensamento de pessoas com tetraplegia, foi a seguinte: "Possibilidade de ampliação da autonomia e compensação da vulnerabilidade a partir da tecnologia assistiva para acesso a computadores".

\section{Discussão}

Verificou-se que as pessoas mais acometidas por lesão medular foram homens, jovens, solteiros e em idade produtiva, mas que passaram a ficar sem ocupação após a lesão. Esse perfil foi confirmado por estudos anteriores e é condizente com a exposição desse grupo a causas externas, especialmente a situações de riscos como acidentes de trânsito, com arma de fogo, quedas de altura e mergulhos em águas rasas (Lee et al.,2014).

Os resultados deste estudo revelaram que as pessoas com lesão medular grave necessitam realizar grandes esforços para superarem as mudanças causadas pela restrição física abrupta a que ficam submetidas, que repercutem fortemente na condição emocional. A sensação de fragilidade, comumente acompanhada de baixa autoestima, levam-as a necessidade de vivenciarem o luto, portanto elas necessitam de suporte emocional para alcançaram a normalização da vida. Isso requer flexibilidade contínua, persistência e soluções focadas no ajustamento, comumente interpretadas processo de resiliência (Geard et al., 2018; Tederko et al., 2017).

Ficou evidente que é necessário trabalhar no processo de recuperação, com o significado global da condição de ser uma pessoa com lesão medular grave, que inclui a interpretação da experiência estressora do momento devido limitação física abrupta, e o estabelecimento de novas ações para o presente, bem como com a oferta de estratégias para planejarem o futuro (Bailey et al., 2016; Liooij et al., 2016).

A TA, portanto, representa a possibilidade de minimização da vulnerabilidade extrema, expressa de forma física, existencial e social (Geard et al., 2018; Tederko et al., 2017; Parimbelli et al., 2017; Folan et al., 2015). Trata-se, pois, de um suporte relevante que ajuda a enfrentar essa nova condição, com serias limitações para as ações e interação cotidianas. Portanto, os serviços de tratamento devem ofertar, tão logo quando forem possíveis, esses recursos, pois representam, além da possibilidade de superar a limitação física iminente, a oportunidade de melhorar a qualidade de vida.

O fato das pessoas com limitações físicas extremas poderem acessar o mundo virtual permite-lhes a sensação de positividade frente à vida, que contribui para o resgate da autonomia, que se refere à capacidade de autodeterminação de um indivíduo (Silva et al., 2021). Possibilita, sobretudo, o resgate da autonomia individual, que está vinculada à liberdade subjetiva, inclui ações ligadas a interesses pessoais e à ausência de coação. Trata-se, pois, da conquista da autonomia privada que abarca a ideia de que o indivíduo deve ser reconhecido como sujeito capaz de deliberar sobre os seus objetivos pessoais (Oviedo \& Czeresnia, 2015).

Notadamente, a conquista da autonomia pressupõe também o princípio lapidar da liberdade de escolha (Oviedo \& Czeresnia, 2015). Os participantes deste estudo revelaram que a TA para acesso ao computador permite escolher onde querem navegar no mundo virtual, o que lhe causa grande sensação de liberdade de escolha. Ressalte-se, na perspectiva da Bioética, o exercício da liberdade de escolha consiste em um direito inalienável da pessoa humana. Portanto, é necessário acionar a 
dimensão protetora da bioética direcionada à redução da vulnerabilidade do indivíduo com tetraplegia e que deve ser conjugada com a responsabilidade dos familiares que dele cuidam e dos profissionais que o assistem (Oviedo \& Czeresnia, 2015; Burwell et al., 2017).

Nessa perspectiva, algumas das representações sobre autonomia demonstrados neste estudo se aproximaram de um conceito mais atual sobre TA, que consiste na interface entre as pessoas com deficiência e seus ambientes exclusivos, viabilizando a interação social (Silva et al., 2021).

O acesso às redes sociais e outras mídias mediado pela TA representa a ampliação da autonomia decisória e executiva. A participação em atividades comunitárias e a possibilidade de contato com o mundo denotam significado coletivo à percepção da autonomia (Burlá, 2014), enfoque que se assemelhou ao de um estudo irlandês, em que usuários de recursos eletrônicos de TA e com lesão medular, foi vinculada à participação (Andrade et al., 2019). Ademais, trazem à baila a perspectiva sóciohistórica da TA, não apenas como um mero instrumento, mas como estratégia inserida num processo social que pode atuar como mediadora do desenvolvimento humano (Andrade et al., 2019).

$\mathrm{O}$ acesso à continuidade do aprendizado, por meio da TA para acesso a computadores, também foi destacado neste estudo. Diversos estudos reiteram a importância do acesso a computadores para que as pessoas com lesão medular grave possam dar continuidade a processos de aprendizagem, não só para crianças em idade escolar, mas para adultos para aprimoramento da capacidade cognitiva e social, que também fica bastante prejudicada (Fabrin et al., 2016; Geard et al., 2018).

O processo de inclusão laboral possibilitado pelos recursos de TA, embora ainda muito insuficiente, foi também destacado pelos participantes deste estudo, sobretudo ao colocar em destaque a necessidade de uma nova perspectiva de educação que modifique o significado e a concepção sobre a inclusão das pessoas com deficiência presente no imaginário social (Andrade et al., 2019).

Ademais, o retorno ao trabalho, por meio do acesso a TA, representou para alguns participantes deste estudo o mais alto grau de autonomia conquistada, pois permitiu o resgate da identidade, proporcionando a alteração na posição social. Contudo, evidenciaram que as oportunidades de emprego ainda são muito escassas, sendo mais acentuadas em cidades menores, onde existem poucas indústrias e serviços públicos que garantam uma parcela das vagas para as pessoas com deficiência física. Além disso, estudos mostram que os ambientes de trabalho não estão adequadamente preparados para incluir as pessoas com deficiências, ou seja, o redimensionamento do espaço físico, do mobiliário, entre outros aspectos (Andrade et al., 2019; Pruettikomon et al., 2018).

Nos últimos anos, embora o Brasil tenha avançado na promoção dos direitos das pessoas com deficiência, por meio de políticas públicas que buscam valorizar a pessoa como cidadã, a agenda governamental ainda é insuficiente para cobrir as demandas (Barbosa, 2016), em particular as exigências relativas ao acesso a TA. Ainda, o reconhecimento do contexto social dessa parcela da população é condição básica necessária para a elaboração de políticas públicas (Barbosa, 2016) e intervenções em saúde, que por sua vez, devem ser pautadas pela discussão bioética desenvolvida no Brasil e na América Latina, mediante seus contrastes sociais e peculiaridades.

Uma das limitações do estudo diz respeito à opção metodológica, um estudo qualitativo que não permite a generalização dos resultados, mas possibilita demonstrar como a TA para acesso a computadores atuam no processo de reconstrução da autonomia das pessoas com lesão medular, que contribui substancialmente para a melhoria da qualidade de vida, pois permite a recuperação da capacidade de se autogovernar, imprescindível para a conquista da dignidade humana.

\section{Considerações Finais}

Os benefícios da TA para acesso a computadores possibilitam a diminuição da vulnerabilidade e a ampliação da autonomia das pessoas portadoras de lesão medular grave, em diversas perspectivas, bem como a sua reinserção social e no 
trabalho. Desta feita, deve-se reconhecer o direito de acesso a TA a essas pessoas para melhoria da qualidade de vida e alcance da dignidade humana. Nesse sentido, os resultados deste estudo demonstram que é necessário incluir nos serviços de atendimento as pessoas com lesão medular grave, desde o inicio do tratamento, a possibilidade de uso TA, com devido acompanhamento de profissionais qualificados para orientar o uso dos mesmos. Assim sendo, torna-se um imperativo ético garantir o acesso dessas pessoas aos recursos de TA, através da ampliação de políticas públicas intersetoriais, que não pode estar apartado de outros esforços empreendidos pelo poder público, no sentido de qualificar a vida social desses cidadãos.

\section{Referências}

Andrade, V. S., Faleiros, F., Balestrero, L. M., Romeiro, V., \& Santos, C. B. (2019). Participação social e autonomia pessoal de indivíduos com lesão medular. Rev Bras Enferm, 72(1), 250-7. doi.org/10.1590/0034-7167-2018-0020

Bailey, K. A., Gammage, K. L., Van Ingen C., \& Ditor D. S. (2016). Managing the stigma: Exploring body image experiences and self-presentation among people with spinal cord injury. Health Psychol.Open, 3(1):1-10. 10.1177/2055102916650094

Balsdassin, V., Lorenzo, C. Shimizu, H. E. (2018). Tecnologia assistiva e qualidade de vida na tetraplegia: abordagem bioética. Rev. Bioét, 26 (4), 574-86. https://doi.org/10.1590/1983-80422018264276

Barbosa, A. (2016). Mobilidade urbana para pessoas com deficiência no Brasil: um estudo em blogs. Rev Bras Gest Urbana, 8(1),142-54.

Beauchamp, T. L., \& Childress, J. F. (2013). Principles of Biomedical Ethics. (7th ed.), Oxford University Press.

Burlá, C., Pessini, L., Siqueira, J. E., \& Nunes, R. Envelhecimento e doença de Alzheimer: reflexões sobre autonomia e o desafio do cuidado. (2014). Rev Bioética, 2014,22(1),85-93. http://www.scielo.br/pdf/bioet/v22n1/a10v22n1.pdf

Charmaz K. (2009). A construção da teoria fundamentada. Guia Prático para análise qualitativa. Editora Artmed.

Decreto $n^{\circ} 10.645$, de 11 de março de 2021. Regulamenta o art. 75 da lei $n^{\circ} 13.146$, de 6 julho de 2015, para dispor sobre as diretrizes, os objetivos e os eixos do plano nacional de tecnologia assistiva. Diário Oficial da União. Brasília, DF. https://www.in.gov.br/en/web/dou/-/decreto-n-10.645-de-11-de-marco-de2021-307923632

Fabrin, A. C. B., Nascimento, P. G. do, \& Fantacini, R. A. F. (2016). Assistive technology: alternative communication for students with cerebral paralysis. Research, Society and Development, 2(2), 136-150. https://doi.org/10.17648/rsd-v2i2.32

Folan, A., Barclay, L., Cooper, C., \& Robinson, M. (2015). Exploring the experience of clients with tetraplegia utilizing assistive technology for computer access. Disabil Rehabil Assist, 10(1), 46-52.

Geard, A., Kirkevold, M., Løvstad, M., \& Schanke, A.K. (2018). Exploring narratives of resilience among seven males living with spinal cord injury: a qualitative study. BMC Psychol, 6(1), 1. 10.1186/s40359-017-0211-2.

Gómara-Toldrà, N., Sliwinski, M., \& Dijkers, M.P. (2014). Physical therapy after spinal cord injury: a systematic review of treatments focused on participation. J Spinal Cord Med, 37(4), 371-9. http://www.pubmedcentral.nih.gov/articlerender.fcgiartid=4116720\&tool=pmcentrez\&rendertype=abstract

Habermas J. (2003). L'éthique de la discussion et la question de la verité. Gasset.

Lee, B.B., Cripps, R.A., Fitzharris, M., Wing, P.C. (2014). The global map for traumatic spinal cord injury epidemiology: update 2011, global incidence rate. Spinal Cord, 52(2), 110-6. 10.1038/sc.2012.158.

Litooij, E., Widdershoven, G. A. M, Stolwijk-Swüste, J. M., Doodeman, S. Leget, C. J., \& Dekker J. (2016). Global meaning in people with spinal cord injury: Content and changes. J Spinal Cord Med, 39(2), 197-205. doi:10.1179/2045772314Y.0000000290

Oviedo, R. A. M., \& Czeresnia, D. (2015). O conceito de vulnerabilidade e seu caráter biossocial. Interface - Comun Saúde, Educ, 19(53), 237-50. http://www.scielo.br/scielo.php?script=sci_arttext\&pid=S1414-32832015000200237\&lng=pt\&nrm=iso\&tlng=en

Parimbelli, E., Pistarini, C., Fizzotti, G., Rognoni, C., Olivieri, G., \& Quaglini, S. (2017). Computer-Assessed Preference-Based Quality of Life in Patients with Spinal Cord Injury. Biomed Res Int. Article ID 4543610. 10.1155/2017/4543610.

Pruettikomon, S., \& Louhapensang, C. (2018). A Study and Development of Workplace Facilities and Working Environment to Increase the Work Efficiency of Persons with Disabilities: A Case Study of Major Retail and Wholesale Companies in Bangkok. The Scientific World Journal, Article ID3142010. $10.1155 / 2018 / 3142010$

Rezende, L. S., Lima, M. B. \& Salvador, E. P. (2018). Interventions for Promoting Physical Activity Among Individuals With Spinal Cord Injury: A Systematic Review. J Phys Act Health, 15(12), 954-59. 10.1123/jpah.2018-0034

Seoane, J. A. (2010). Las autonomías del paciente. Dilemata, 3:61-75.

Schwartz, C. E., Stucky, B., Rivers, C. S., Noonan, V. K., Finkelstein, J. A. \& RHSCIR Network. (2018). Quality of life and adaptation in people with spinal cord injury: response rhift effects from 1 to 5 years postinjury. Arch Phys Med Rehabil, 99(8),1599-1608. 10.1016/j.apmr.2018.01.028. PMID: 29481771.

Silva, H. F. da., Silva, J. F. da., Cruz, V. V., Figueiredo, N. M. A. de., Sé, A. C. S., \& Machado, W. C. A. (2021). Applicability of Assistive Technology for 
Research, Society and Development, v. 11, n. 2, e9711219793, 2022

(CC BY 4.0) | ISSN 2525-3409 | DOI: http://dx.doi.org/10.33448/rsd-v11i2.19793

people with spinal cord injury: An integrative review. Research, Society and Development, 10(8), e0210815387.

Tederko, P., Krasuski, T., Krasuski, M., Długołęcka, A., \& Tarnacka, B. (2017). Determinants of health knowledge and health perceptions from the perspective of health-related education of patients with spinal cord injury: a systematic review. Int J Rehabil Res., 40(2), 97-106. 10.1097/MRR.0000000000000215.

United Nations. (2011). Disability and the Millennium Development Goals. http://www.un.org/disabilities/documents/review_of_disability_and_the_mdgs.pdf

Unesco. (2005). Declaração Universal Sobre Bioética e Direitos Humanos. Tradução: Cátedra Unesco de Bioética da Universidade de Brasília. Disponível em http://fs.unb.br/images/Pdfs/Bioetica/DUBDH.pdf 